\title{
Symmetry Lie algebra and exact solutions of some fourth-order difference equations
}

\author{
N. Mnguni, D. Nyirenda, M. Folly-Gbetoula*
}

School of Mathematics, University of the Witwatersrand, 2050, Johannesburg, South Africa.

Communicated by C. Park

\section{Abstract}

In this paper, all the Lie point symmetries of difference equations of the form

$$
u_{n+4}=\frac{u_{n}}{A_{n}+B_{n} u_{n} u_{n+2}}
$$

where, $\left(A_{n}\right)_{n \geqslant 0}$ and $\left(B_{n}\right)_{n \geqslant 0}$ are sequences of real numbers, are obtained. We perform reduction of order using the invariant of the group of transformations. Furthermore, we obtain their solutions. In particular, our work generalizes some results in the literature.

Keywords: Difference equation, symmetry, group invariant solutions.

2010 MSC: 39A10, 39A99, 39A13.

(C)2018 All rights reserved.

\section{Introduction}

Lie symmetry analysis is a powerful method not only for differential equations but also difference equations. Its demonstration on difference equations has been successful and there has been progress in this area (see $[16,19,20])$. The symmetry method has been used to find traveling wave solutions. For more on traveling waves, refer to [12, 21-23].

In this method, one finds a group of mappings that map the set of solutions to the difference equation under study onto itself. However, it should be stated that the computational difficulty in employing this method can increase with increasing order of the equation being studied.

Elsayed [9] obtained the exact solutions of

$$
x_{n+1}=\frac{x_{n-3}}{ \pm 1 \pm x_{n-1} x_{n-3}}
$$

\footnotetext{
${ }^{*}$ Corresponding author

Email addresses: 734552@students.wits.ac.za (N. Mnguni), Darlison.Nyirenda@wits.ac.za (D. Nyirenda), Mensah.Folly-Gbetoula@wits.ac.za (M. Folly-Gbetoula)
}

doi: $10.22436 /$ jnsa.011.11.06

Received: 2017-10-30 Revised: 2018-08-04 Accepted: 2018-08-07 
Related work has been done (see $[1-11,13-15,17,18])$. In this paper we obtain solutions of the following difference equations via the invariant of their group of transformations:

$$
x_{n+1}=\frac{x_{n-3}}{a_{n}+b_{n} x_{n-1} x_{n-3}}
$$

for some arbitrary sequences of real numbers $\left(a_{n}\right)$ and $\left(b_{n}\right)$. Thus the solution to the difference equation above extends the solution of Elsayed [9] to equation (1.1) to a more general setting.

Without loss of generality, we instead study the difference equation

$$
u_{n+4}=\frac{u_{n}}{A_{n}+B_{n} u_{n} u_{n+2}}
$$

\subsection{Preliminaries}

Consider the difference equation

$$
u_{n+4}=f\left(n, u_{n}, u_{n+1}, \ldots, u_{n+3}\right),
$$

where $f$ is an arbitrary function. Suppose that the point transformations are of the form

$$
\left(n, u_{n}\right) \mapsto\left(n, u_{n}+\epsilon Q\left(n, u_{n}\right)\right),
$$

where $\mathrm{Q}$ is the characteristic and

$$
X=Q\left(n, u_{n}\right) \frac{\partial}{\partial u_{n}}+Q\left(n+1, u_{n+1}\right) \frac{\partial}{\partial u_{n+1}}+\cdots+Q\left(n+3, u_{n+3}\right) \frac{\partial}{\partial u_{n+3}}
$$

is the corresponding symmetry generator. We have the following linearized symmetry condition, obtained using (1.3):

$$
Q\left(n+4, u_{n+4}\right)-\frac{\partial f}{\partial u_{n}} Q\left(n, u_{n}\right)-\frac{\partial f}{\partial u_{n+2}} Q\left(n+2, u_{n+2}\right)-\frac{\partial f}{\partial u_{n+3}} Q\left(n+3, u_{n+3}\right)=0,
$$

whenever (1.2) holds. In solving our difference equation under study, we will use a canonical coordinate, that is the variable $S$ such that

$$
\mathrm{XS}=1 .
$$

We make use of the known choice of $S$ given by (see [16])

$$
S=\int \frac{d u_{n}}{Q\left(n, u_{n}\right)}
$$

Equation (1.4) appears simpler, although it is generally hard to solve.

\section{Main results}

We apply the symmetry condition (1.4) to

$$
u_{n+4}=\frac{u_{n}}{A_{n}+B_{n} u_{n} u_{n+2}}
$$

to solve for the characteristic $\mathrm{Q}$. This yields

$$
Q\left(n+4, u_{n+4}\right)+\frac{B_{n} u_{n}{ }^{2}}{\left(B_{n} u_{n} u_{n+2}+A_{n}\right)^{2}} Q\left(n+2, u_{n+2}\right)-\frac{A_{n}}{\left(B_{n} u_{n} u_{n+2}+A_{n}\right)^{2}} Q\left(n, u_{n}\right)
$$


In oder to get an equation that involves $u_{n}$ only, we first differentiate implicitly (2.2) with respect to $u_{n}$ (keeping $u_{n+4}$ fixed). This results in

$$
\frac{\partial}{\partial u_{n+2}} Q\left(n+2, u_{n+2}\right)-\frac{\partial}{\partial u_{n}} Q\left(n, u_{n}\right)+\frac{2}{u_{n}} Q\left(n, u_{n}\right)=0 .
$$

Secondly, we differentiate (2.3) with respect to $u_{n}$. This yields

$$
\frac{d}{d u_{n}}\left(-\frac{\partial}{\partial u_{n}} Q\left(n, u_{n}\right)+\frac{2}{u_{n}} Q\left(n, u_{n}\right)\right)=0
$$

So,

$$
-\frac{\partial}{\partial u_{n}} Q\left(n, u_{n}\right)+\frac{2}{u_{n}} Q\left(n, u_{n}\right)=f_{2}(n)
$$

Thus Q takes the form

$$
Q\left(n, u_{n}\right)=f_{1}(n) u_{n}{ }^{2}+f_{2}(n) u_{n}
$$

where $f_{1}$ and $f_{2}$ are functions of $n$. Finally, we substitute (2.4) into (2.2) and do the separation by powers of shifts of $u_{n}$. This yields a system of equations that simplifies to

$$
\left\{\begin{array}{l}
f_{1}(n)=0 \\
f_{2}(n)+f_{2}(n+2)=0 .
\end{array}\right.
$$

Hence,

$$
f_{1}(n)=0, f_{2}(n)=\alpha^{n}, f_{2}(n)=\bar{\alpha}^{n},
$$

where $\alpha=\exp \{\mathfrak{i} \pi / 2\}$ and $\bar{\alpha}$ is the complex conjugate. Hence, we have two generators given as follows:

$$
\begin{aligned}
& x_{0}=\alpha^{n} u_{n} \partial_{u_{n}}+\alpha^{n+1} u_{n+1} \partial_{u_{n+1}}+\alpha^{n+2} u_{n+2} \partial_{u_{n+2}}+\alpha^{n+3} u_{n+3} \partial_{u_{n+3}}, \\
& x_{1}=\bar{\alpha}^{n} u_{n} \partial_{u_{n}}+\bar{\alpha}^{n+1} u_{n+1} \partial_{u_{n+1}}+\bar{\alpha}^{n+2} u_{n+2} \partial_{u_{n+2}}+\bar{\alpha}^{n+3} u_{n+3} \partial_{u_{n+3}} .
\end{aligned}
$$

Let

$$
S_{n}=\int \frac{d u_{n}}{\alpha^{n} u_{n}}=\frac{1}{\alpha^{n}} \ln \left|u_{n}\right|
$$

We perform a change of variable, thanks to (2.5):

$$
\tilde{V}_{n}=S_{n} \alpha^{n}+S_{n+2} \alpha^{n+2} .
$$

We have

$$
X_{0} \tilde{V}_{n}=X_{1} \tilde{V}_{n}=0
$$

so that $\tilde{V}_{n}$ is an invariant of the group of transformations (1.3). Considering the fact that the equation being studied is rational, it is convenient to utilise

$$
\left|V_{n}\right|=\exp \left\{-\tilde{V}_{n}\right\}
$$

Using $V_{n}$ with (2.1), we obtain

$$
V_{n+2}=A_{n} V_{n} \pm B_{n}
$$


Now we make the choice of using the plus sign and thus write the solution of (2.6) as

$$
V_{2 n+i}=V_{i}\left(\prod_{k_{1}=0}^{n-1} A_{2 k_{1}+i}\right)+\sum_{l=0}^{n-1}\left(B_{2 l+i} \prod_{k_{2}=l+1}^{n-1} A_{2 k_{2}+i}\right),
$$

where $i=0,1$. Thus the solution of (2.1) can be obtained by reversing the changes of variables. We have

$$
\begin{aligned}
\left|u_{n}\right|=\exp \left(\alpha_{n} s_{n}\right) & =\exp \left(\alpha^{n} c_{1}+\bar{\alpha}^{n} c_{2}+\frac{1}{2} \sum_{k_{1}=0}^{n-1} \alpha^{n} \bar{\alpha}^{k_{1}} \ln \left|V_{k_{1}}\right|+\frac{1}{2} \sum_{k_{2}=0}^{n-1} \bar{\alpha}^{n} \alpha^{k_{2}} \ln \left|V_{k_{2}}\right|\right) \\
& =\exp \left(\alpha^{n} c_{1}+\bar{\alpha}^{n} c_{2}+\frac{1}{2} \sum_{k=0}^{n-1}\left(\alpha^{n-k}+\bar{\alpha}^{n-k}\right) \ln \left|V_{k}\right|\right) \\
& =\exp \left(\alpha^{n} c_{1}+\bar{\alpha}^{n} c_{2}+\sum_{k=0}^{n-1} \operatorname{Re}\left(\alpha^{n-k}\right) \ln \left|V_{k}\right|\right) \\
& =\exp \left(\alpha^{n} c_{1}+\bar{\alpha}^{n} c_{2}+\sum_{k=0}^{n-1} \cos \left(\frac{(n-k) \pi}{2}\right) \ln \left|V_{k}\right|\right) .
\end{aligned}
$$

Setting $n:=4 n+j$ and $H_{j}=\alpha^{j} c_{1}+\bar{\alpha}^{j} c_{2}$, we obtain

$$
\left|u_{4 n+j}\right|=\exp \left(H_{j}+\sum_{k=0}^{4 n+j-1}\left(\cos \left(\frac{(j-k) \pi}{2}\right)\right) \ln \left|V_{k}\right|\right) .
$$

For $j=0,(2.8)$ becomes

$$
\left|\mathrm{u}_{4 n}\right|=\exp \left(\mathrm{H}_{0}\right) \exp \left(\ln \left|\mathrm{V}_{0}\right|-\ln \left|\mathrm{V}_{2}\right|+\ln \left|\mathrm{V}_{4}\right|-\ln \left|\mathrm{V}_{6}\right|+\cdots+\ln \left|\mathrm{V}_{4 n-4}\right|-\ln \left|\mathrm{V}_{4 n-2}\right|\right) .
$$

However, using (2.8) with $j=1, n=0,\left|u_{0}\right|=\exp \left(\mathrm{H}_{0}\right)$. It can be shown that we do not need the absolute values, thus

$$
u_{4 n}=u_{0} \prod_{s=0}^{n-1} \frac{v_{4 s}}{v_{4 s+2}}
$$

Using (2.7), where $n:=2 s$ and $i=0$ for $V_{4 s}$, and $n:=2 s+1$ and $i=0$ for $V_{4 s+2}$, we have

$$
\begin{aligned}
\mathrm{V}_{4 s}=\mathrm{V}_{0} \prod_{\mathrm{k}_{1}=0}^{2 s-1} A_{2 \mathrm{k}_{1}}+\sum_{\mathrm{l}=0}^{2 s-1} \mathrm{~B}_{2 l} \prod_{\mathrm{k}_{2}=l+1}^{2 s-1} A_{2 \mathrm{k}_{2}} & =\mathrm{V}_{0}\left(\prod_{\mathrm{k}_{1}=0}^{2 s-1} A_{2 \mathrm{k}_{1}}+\frac{1}{\mathrm{~V}_{0}} \sum_{l=0}^{2 s-1} B_{2 l} \prod_{k_{2}=l+1}^{2 s-1} A_{2 \mathrm{k}_{2}}\right) \\
& =\frac{1}{u_{0} u_{2}}\left(\prod_{k_{1}=0}^{2 s-1} A_{2 k_{1}}+u_{0} u_{2} \sum_{l=0}^{2 s-1} B_{2 l} \prod_{k_{2}=l+1}^{2 s-1} A_{2 k_{2}}\right),
\end{aligned}
$$

and

$$
\begin{aligned}
\mathrm{v}_{4 s+2}=\mathrm{v}_{0} \prod_{\mathrm{k}_{1}=0}^{2 s} A_{3 k_{1}}+\sum_{\mathrm{l}=0}^{2 s} B_{3 l} \prod_{\mathrm{k}_{2}=\mathrm{l}+1}^{2 s} A_{3 k_{2}} & =v_{0}\left(\prod_{k_{1}=0}^{2 s} A_{2 k_{1}}+\frac{1}{V_{0}} \sum_{l=0}^{2 s} B_{2 l} \prod_{k_{2}=l+1}^{2 s} A_{2 k_{2}}\right) \\
& =\frac{1}{u_{0} u_{2}}\left(\prod_{k_{1}=0}^{2 s} A_{2 k_{1}}+u_{0} u_{2} \sum_{l=0}^{2 s} B_{2 l} \prod_{k_{2}=l+1}^{2 s} A_{2 k_{2}}\right) .
\end{aligned}
$$


Thus

$$
u_{4 n}=u_{0} \prod_{s=0}^{n-1} \frac{\prod_{k_{1}=0}^{2 s-1} A_{2 k_{1}}+u_{0} u_{2} \sum_{l=0}^{2 s-1} B_{2 l} \prod_{k_{1}=0}^{2 s-1} A_{2 k_{1}}+u_{0} u_{2} \sum_{l=0}^{2 s} B_{2 l} A_{k_{2}=l+1}^{2 s} A_{2 k_{2}}}{\prod_{k_{2}}{ }_{k}}
$$

which implies that

$$
x_{4 n-3}=x_{-3} \prod_{s=0}^{n-1} \frac{\prod_{k_{1}=0}^{2 s-1} a_{2 k_{1}}+x_{-3} x_{-1} \sum_{l=0}^{2 s-1} b_{2 l} \prod_{k_{2}=l+1}^{2 s-1} a_{2 k_{2}} a_{2 k_{1}}+x_{-3} x_{-1} \sum_{l=0}^{2 s} b_{2 l} \prod_{k_{2}=l+1}^{2 s} a_{2 k_{2}}}{\prod_{k}{ }_{k}}
$$

For $\mathfrak{j}=1$, (2.8) becomes

$$
\left|\mathrm{u}_{4 \mathrm{n}+1}\right|=\exp \left(\mathrm{H}_{1}\right) \exp \left(\ln \left|\mathrm{V}_{1}\right|-\ln \left|\mathrm{V}_{3}\right|+\ln \left|\mathrm{V}_{5}\right|-\ln \left|\mathrm{V}_{7}\right|+\ldots+\ln \left|\mathrm{V}_{4 \mathrm{n}-3}\right|-\ln \left|\mathrm{V}_{4 \mathrm{n}-1}\right|\right) .
$$

However, using (2.8) with $j=1, n=0,\left|u_{1}\right|=\exp \left(\mathrm{H}_{1}\right)$. It can be shown that we do not need the absolute values, thus

$$
u_{4 n+1}=u_{1} \prod_{s=0}^{n-1} \frac{v_{4 s+1}}{v_{4 s+3}}
$$

By (2.7), we have

$$
\begin{aligned}
\mathrm{V}_{4 s+1} & =\mathrm{V}_{1} \prod_{\mathrm{k}_{1}=0}^{2 s-1} A_{2 \mathrm{k}_{1}+1}+\sum_{\mathrm{l}=0}^{2 s-1} \mathrm{~B}_{2 \mathrm{l}+1} \prod_{\mathrm{k}_{2}=\mathrm{l}+1}^{2 s-1} \mathrm{~A}_{2 \mathrm{k}_{2}+1} \\
& =\mathrm{V}_{1}\left(\prod_{\mathrm{k}_{1}=0}^{2 s-1} A_{2 \mathrm{k}_{1}+1}+\frac{1}{\mathrm{~V}_{1}} \sum_{\mathrm{l}=0}^{2 s-1} \mathrm{~B}_{2 \mathrm{l}+1} \prod_{\mathrm{k}_{2}=\mathrm{l}+1}^{2 s-1} \mathrm{~A}_{2 \mathrm{k}_{2}}\right) \\
& =\frac{1}{\mathrm{u}_{1} \mathrm{u}_{3}}\left(\prod_{\mathrm{k}_{1}=0}^{2 s-1} A_{2 \mathrm{k}_{1}+1}+\mathrm{u}_{1} \mathrm{u}_{3} \sum_{\mathrm{l}=0}^{2 s-1} \mathrm{~B}_{2 \mathrm{l}+1} \prod_{\mathrm{k}_{2}=\mathrm{l}+1}^{2 s-1} A_{2 \mathrm{k}_{2}+1}\right),
\end{aligned}
$$

and similarly,

$$
v_{4 s+3}=\frac{1}{u_{1} u_{5}}\left(\prod_{k_{1}=0}^{2 s} A_{2 k_{1}+1}+u_{1} u_{3} \sum_{l=0}^{2 s} B_{2 l+1} \prod_{k_{2}=l+1}^{2 s} A_{2 k_{2}+1}\right)
$$

Now

$$
u_{4 n+1}=u_{1} \prod_{s=0}^{n-1} \frac{\prod_{k_{1}=0}^{2 s-1} A_{2 k_{1}+1}+u_{1} u_{3} \sum_{l=0}^{2 s-1} B_{2 l+1} \prod_{k_{2}=l+1}^{2 s-1} A_{2 k_{2}+1} A_{2 k_{1}+1}+u_{1} u_{3} \sum_{l=0}^{2 s} B_{2 l+1} \prod_{k_{2}=l+1}^{2 s} A_{2 k_{2}+1}}{\prod_{l=0}}
$$

which implies that

$$
x_{4 n-2}=x_{-2} \prod_{s=0}^{n-1} \frac{\prod_{k_{1}=0}^{2 s-1} a_{2 k_{1}+1}+x_{-2} x_{0} \sum_{l=0}^{2 s-1} b_{2 l+1} \prod_{k_{2}=l+1}^{2 s-1} a_{2 k_{2}+1} a_{2 k_{1}+1}+x_{-2} x_{0} \sum_{l=0}^{2 s} b_{2 l+1} \prod_{k_{2}=l+1}^{2 s} a_{2 k_{2}+1}}{\prod_{l=0}{ }_{k}}
$$

For $j=2$, we find that (2.8) becomes

$$
\left|\mathfrak{u}_{4 n+2}\right|=\exp \left(\mathrm{H}_{2}\right) \exp \left(-\ln \left|\mathrm{V}_{0}\right|+\ln \left|\mathrm{V}_{2}\right|-\ln \left|\mathrm{V}_{4}\right|+\cdots+\ln \left|\mathrm{V}_{4 n-2}\right|-\ln \left|\mathrm{V}_{4 n}\right|\right) .
$$


Similar to the earlier cases, setting $n=0$ and $j=2$ yields the equation $\left|u_{2}\right|=\exp \left(H_{2}\right) \frac{1}{\left|V_{0}\right|}$. So we have

$$
u_{4 n+2}=u_{2} \prod_{s=0}^{n-1} \frac{v_{4 s+2}}{v_{4 s+4}}
$$

The expressions for $V_{4 s+2}$ and $V_{4 s+4}$ are obtained from (2.7), by setting $n=2 s+1, i=0$ and $n=2 s+2, i=$ 0 , respectively. They are as follows:

$$
\begin{aligned}
& V_{4 s+2}=v_{0} \prod_{k_{1}=0}^{2 s} A_{2 k_{1}}+\sum_{l=0}^{2 s} B_{2 l} \prod_{k_{2}=l+1}^{2 s} A_{2 k_{2}}=V_{0}\left(\prod_{k_{1}=0}^{2 s} A_{2 k_{1}}+\frac{1}{V_{0}} \sum_{l=0}^{2 s} B_{2 l} \prod_{k_{2}=l+1}^{2 s} A_{2 k_{2}}\right) \\
& =\frac{1}{u_{0} u_{2}}\left(\prod_{k_{1}=0}^{2 s} A_{2 k_{1}}+u_{0} u_{2} \sum_{l=0}^{2 s} B_{2 l} \prod_{k_{2}=l+1}^{2 s} A_{2 k_{2}}\right) \text {, }
\end{aligned}
$$

and

$$
\begin{aligned}
& V_{4 s+4}=V_{0} \prod_{k_{1}=0}^{2 s+1} A_{2 k_{1}}+\sum_{l=0}^{2 s+1} B_{2 l} \prod_{k_{2}=l+1}^{2 s+1} A_{2 k_{2}}=V_{0}\left(\prod_{k_{1}=0}^{2 s+1} A_{2 k_{1}}+\frac{1}{V_{0}} \sum_{l=0}^{2 s+1} B_{2 l} \prod_{k_{2}=l+1}^{2 s=1} A_{2 k_{2}}\right) \\
& =\frac{1}{u_{0} u_{2}}\left(\prod_{k_{1}=0}^{2 s+1} A_{2 k_{1}}+u_{0} u_{2} \sum_{l=0}^{2 s+1} B_{2 l} \prod_{k_{2}=l+1}^{2 s+1} A_{2 k_{2}}\right) \text {. }
\end{aligned}
$$

Hence

$$
u_{4 n+2}=u_{2} \prod_{s=0}^{n-1} \frac{\prod_{k_{1}=0}^{2 s} A_{2 k_{1}}+u_{0} u_{2} \sum_{l=0}^{2 s} B_{2 l} \prod_{k_{1}=0}^{2 s} A_{2 k_{1}}+u_{0} u_{2} \sum_{l=0}^{2 s+1} B_{2 l} A_{2 k_{2}} \prod_{k_{2}=l+1}^{2 s+1} A_{2 k_{2}}}{2 s+1}
$$

which gives

$$
x_{4 n-1}=x_{-1} \prod_{s=0}^{n-1} \frac{\prod_{k_{1}=0}^{2 s} a_{2 k_{1}}+x_{-3} x_{-1} \sum_{l=0}^{2 s} b_{2 l} \prod_{k_{1}=0}^{2 s} a_{2 k_{1}}+x_{-3} x_{-1} \sum_{l=0}^{2 s+1} b_{2 l} \prod_{k_{2}=l+1}^{2 s+1} a_{2 k_{2}}}{2 s+1} a_{l=1}
$$

For $\mathfrak{j}=3,(2.8)$ becomes

$$
\left|\mathrm{u}_{4 \mathrm{n}+3}\right|=\exp \left(\mathrm{H}_{3}\right) \exp \left(-\ln \left|\mathrm{V}_{1}\right|+\ln \left|\mathrm{V}_{3}\right|-\ln \left|\mathrm{V}_{5}\right|+\cdots+\ln \left|\mathrm{V}_{4 \mathrm{n}-1}\right|-\ln \left|\mathrm{V}_{4 \mathrm{n}+1}\right|\right) .
$$

Setting $n=0$ and $j=3$, we find that $\left|u_{3}\right|=\exp \left(H_{3}\right) \frac{1}{\left|V_{1}\right|}$. Hence $u_{4 n+3}=u_{3} \prod_{s=1}^{n-1} \frac{V_{4 s+3}}{V_{4 s+5}}$. Following a similar approach as was done in the earlier cases $(j=0,1,2)$, the reader can verify that

$$
u_{4 n+3}=u_{3} \prod_{s=0}^{n-1} \frac{\prod_{k_{1}=0}^{2 s} A_{2 k_{1}+1}+u_{1} u_{3} \sum_{l=0}^{2 s} B_{2 l+1} \prod_{k_{2}=l+1}^{2 s} A_{2 k_{2}+1} A_{2 k_{1}+1}+u_{1} u_{3} \sum_{l=0}^{2 s+1} B_{2 l+1} \prod_{k_{2}=l+1}^{2 s+1} A_{2 k_{2}+1}}{2 s+1}
$$

Thus

$$
x_{4 n}=x_{0} \prod_{s=0}^{n-1} \frac{\prod_{k_{1}=0}^{2 s} a_{2 k_{1}+1}+x_{-2} x_{0} \sum_{l=0}^{2 s} b_{2 l+1} \prod_{k_{1}=0}^{2 s+1} a_{2 k_{1}+1}+x_{-2} x_{0} \sum_{l=0}^{2 s+1} b_{2 l+1} a_{2 k_{2}+1} \prod_{k_{2}=l+1}^{2 s+1} a_{2 k_{2}+1}}{2 s+1}
$$


Therefore, the solution $\left\{x_{n}\right\}$ to the equation

$$
x_{n+1}=\frac{x_{n-3}}{a_{n}+b_{n} x_{n-1} x_{n-3}}
$$

satisfies equations (2.9), (2.10), (2.11), and (2.12), as long as the denominators do not vanish.

\section{The case when $a_{j}$ and $b_{j}$ are 2-periodic sequences}

We assume that $\left\{a_{j}\right\}_{j} \geqslant 0=\left\{a_{0}, a_{1}, a_{0}, \ldots\right\}$ where $a_{0} \neq a_{1}$, and $\left\{b_{j}\right\}_{j} \geqslant 0=\left\{b_{0}, b_{1}, b_{0}, b_{1}, \ldots\right\}$ where $b_{0} \neq b_{1}$.

3.1. $a_{0} \neq 1, a_{1} \neq 1$

Then after substitution, (2.9), (2.10), (2.11), and (2.12) become

$$
\begin{aligned}
x_{4 n-3}=x_{-3} \prod_{s=0}^{n-1} \frac{a_{0}^{2 s}+x_{-3} x_{-1} b_{0} \frac{1-a_{0}^{2 s}}{1-a_{0}}}{a_{0}^{2 s+1}+x_{-3} x_{-1} b_{0} \frac{1-a_{0}^{2 s+1}}{1-a_{0}}}, & x_{4 n-2}=x_{-2} \prod_{s=0}^{n-1} \frac{a_{1}^{2 s}+x_{-2} x_{0} b_{1} \frac{1-a_{1}^{2 s}}{1-a_{1}}}{a_{1}^{2 s+1}+x_{-2} x_{0} b_{1} \frac{1-a_{1}^{2 s+1}}{1-a_{1}}}, \\
x_{4 n-1}=x_{-1} \prod_{s=0}^{n-1} \frac{a_{0}^{2 s+1}+x_{-3} x_{-1} b_{0} \frac{1-a_{0}^{2 s+1}}{1-a_{0}}}{a_{0}^{2 s+2}+x_{-3} x_{-1} b_{0} \frac{1-a_{0}^{2 s}}{1-a_{0}}}, & x_{4 n}=x_{0} \prod_{s=0}^{n-1} \frac{a_{1}^{2 s+1}+x_{-2} x_{0} b_{1} \frac{1-a_{1}^{2 s+1}}{1-a_{1}}}{a_{1}^{2 s+2}+x_{-2} x_{0} b_{1} \frac{1-a_{1}^{2 s+2}}{1-a_{1}}}
\end{aligned}
$$

where

$$
\prod_{i=1}^{2}\left(\frac{b_{0}\left(1-a_{0}^{2 s+i}\right)}{1-a_{0}} x_{-3} x_{-1}+a_{0}^{2 s+i}\right)\left(\frac{b_{1}\left(1-a_{1}^{2 s+i}\right)}{1-a_{1}} x_{-2} x_{0}+a_{1}^{2 s+i}\right) \neq 0
$$

for all $s=1,2, \ldots, n-1$.

\section{The case when $a_{j}$ and $b_{j}$ are 1-periodic}

In this case, replace $a_{1}$ and $b_{1}$, in the above section, by $a_{0}$ and $b_{0}$, respectively.

\section{1. $\mathrm{a}_{0} \neq 1$}

In this case, the solution equations are given by

$$
\begin{array}{lr}
x_{4 n-3}=x_{-3} \prod_{s=0}^{n-1} \frac{a_{0}^{2 s}+x_{-3} x_{-1} b_{0} \frac{1-a_{0}^{2 s}}{1-a_{0}}}{a_{0}^{2 s+1}+x_{-3} x_{-1} b_{0} \frac{1-a_{0}^{2 s+1}}{1-a_{0}}}, & x_{4 n-2}=x_{-2} \prod_{s=0}^{n-1} \frac{a_{0}^{2 s}+x_{-2} x_{0} b_{0} \frac{1-a_{0}^{2 s}}{1-a_{0}}}{a_{0}^{2 s+1}+x_{-2} x_{0} b_{0} \frac{1-a_{0}^{2 s+1}}{1-a_{0}}}, \\
x_{4 n-1}=x_{-1} \prod_{s=0}^{n-1} \frac{a_{0}^{2 s+1}+x_{-3} x_{-1} b_{0} \frac{1-a_{0}^{2 s+1}}{1-a_{0}}}{a_{0}^{2 s+2}+x_{-3} x_{-1} b_{0} \frac{1-a_{0}^{2 s+2}}{1-a_{0}}}, \quad x_{4 n}=x_{0} \prod_{s=0}^{n-1} \frac{a_{0}^{2 s+1}+x_{-2} x_{0} b_{0} \frac{1-a_{0}^{2 s+1}}{1-a_{0}}}{a_{0}^{2 s+2}+x_{-2} x_{0} b_{0} \frac{1-a_{0}^{2 s+2}}{1-a_{0}}},
\end{array}
$$

where

$$
\prod_{i=1}^{2}\left(\frac{b_{0}\left(1-a_{0}^{2 s+i}\right)}{1-a_{0}} x_{-3} x_{-1}+a_{0}^{2 s+i}\right)\left(\frac{b_{0}\left(1-a_{0}^{2 s+i}\right)}{1-a_{0}} x_{-2} x_{0}+a_{0}^{2 s+i}\right) \neq 0
$$

for all $\mathrm{s}=1,2, \ldots, \mathrm{n}-1$.

\subsection{1. $\mathrm{a}_{0}=-1, \mathrm{~b}_{0}=1$}

The solution is given by the following equations, and it appears in [9, Theorem 6].

$$
\begin{aligned}
x_{4 n-3} & =x_{-3}\left(-1+x_{-3} x_{-1}\right)^{-n}, & x_{4 n-2} & =x_{-2}\left(-1+x_{-2} x_{0}\right)^{-n}, \\
x_{4 n-1} & =x_{-1}\left(-1+x_{-3} x_{-1}\right)^{n}, & x_{4 n} & =x_{0}\left(-1+x_{-2} x_{0}\right)^{n},
\end{aligned}
$$

where $x_{-2} x_{0} \neq 1$ and $x_{-3} x_{-1} \neq 1$. 


\subsection{2. $\mathrm{a}_{0}=-1, \mathrm{~b}_{0}=-1$}

The solution is given by the following equations, and it appears in [9, Theorem 9].

$$
\begin{aligned}
& x_{4 n-3}=(-1)^{n} x_{-3}\left(1+x_{-3} x_{-1}\right)^{-n}, \\
& x_{4 n-1}=(-1)^{n} x_{-1}\left(1+x_{-3} x_{-1}\right)^{n},
\end{aligned}
$$

$$
\begin{aligned}
x_{4 n-2} & =(-1)^{n} \chi_{-2}\left(1+x_{-2} x_{0}\right)^{-n}, \\
x_{4 n} & =(-1)^{n} \chi_{0}\left(1+x_{-2} x_{0}\right)^{n},
\end{aligned}
$$

where $x_{-2} x_{0} \neq-1$ and $x_{-3} x_{-1} \neq-1$.

4.2. $\mathrm{a}_{0}=1$

The solution is given by the following equations:

$$
\begin{aligned}
x_{4 n-3} & =x_{-3} \prod_{s=0}^{n-1} \frac{1+2 s b_{0} x_{-3} x_{-1}}{1+(2 s+1) b_{0} x_{-3} x_{-1}}, & x_{4 n-2} & =x_{-2} \prod_{s=0}^{n-1} \frac{1+2 s b_{0} x_{-2} x_{0}}{1+(2 s+1) b_{0} x_{-2} x_{0}}, \\
x_{4 n-1} & =x_{-1} \prod_{s=0}^{n-1} \frac{1+(2 s+1) b_{0} x_{-3} x_{-1}}{1+(2 s+2) b_{0} x_{-3} x_{-1}}, & x_{4 n} & =x_{0} \prod_{s=0}^{n-1} \frac{1+(2 s+1) b_{0} x_{-2} x_{0}}{1+(2 s+2) b_{0} x_{-2} x_{0}},
\end{aligned}
$$

where $j b_{0} x_{-3} x_{-1} \neq-1$ and $j b_{0} x_{-2} x_{0} \neq-1$ for all $j=1,2, \ldots, 2 n$.

4.2.1. $\mathrm{a}_{0}=1, \mathrm{~b}_{0}=1$

The solution is given by the following equations, and it appears in [9, Theorem 1]:

$$
\begin{aligned}
x_{4 n-3} & =x_{-3} \prod_{s=0}^{n-1} \frac{1+2 s x_{-3} x_{-1}}{1+(2 s+1) x_{-3} x_{-1}}, & x_{4 n-2} & =x_{-2} \prod_{s=0}^{n-1} \frac{1+2 s x_{-2} x_{0}}{1+(2 s+1) x_{-2} x_{0}}, \\
x_{4 n-1} & =x_{-1} \prod_{s=0}^{n-1} \frac{1+(2 s+1) x_{-3} x_{-1}}{1+(2 s+2) x_{-3} x_{-1}}, & x_{4 n} & =x_{0} \prod_{s=0}^{n-1} \frac{1+(2 s+1) x_{-2} x_{0}}{1+(2 s+2) x_{-2} x_{0}},
\end{aligned}
$$

where $j x_{-3} x_{-1} \neq-1$ and $j x_{-2} x_{0} \neq-1$ for all $j=1,2, \ldots, 2 n$.

\subsection{2. $\mathrm{a}_{0}=1, \mathrm{~b}_{0}=-1$}

The solution is given by the following equations, and it appears in [9, Theorem 4]:

$$
\begin{array}{rlrl}
\chi_{4 n-3} & =x_{-3} \prod_{s=0}^{n-1} \frac{1-2 s x_{-3} x_{-1}}{1-(2 s+1) x_{-3} x_{-1}}, & x_{4 n-2} & =x_{-2} \prod_{s=0}^{n-1} \frac{1-2 s x_{-2} x_{0}}{1-(2 s+1) x_{-2} x_{0}}, \\
x_{4 n-1} & =x_{-1} \prod_{s=0}^{n-1} \frac{1-(2 s+1) x_{-3} x_{-1}}{1-(2 s+2) x_{-3} x_{-1}}, & x_{4 n}=x_{0} \prod_{s=0}^{n-1} \frac{1-(2 s+1) x_{-2} x_{0}}{1-(2 s+2) x_{-2} x_{0}}
\end{array}
$$

where $j x_{-3} x_{-1} \neq 1$ and $j x_{-2} x_{0} \neq 1$ for all $j=1,2, \ldots, 2 n$.

\section{Conclusion}

We obtained two non-trivial symmetry generators of shifted equation and used one of these generators to obtain exact solutions to difference equations of the form

$$
x_{n+1}=\frac{x_{n-3}}{a_{n}+b_{n} x_{n-1} x_{n-3}},
$$

where $\left(a_{n}\right)$ and $\left(b_{n}\right)$ are arbitrary sequences of real numbers. In particular, our work generalized a result of Elsayed [9]. 


\section{Acknowledgment}

We would like to thank the reviewers for their fruitful suggestions that helped improve the paper.

\section{References}

[1] M. Aloqeli, Dynamics of a rational difference equation, Appl. Math. Comput., 176 (2006), 768-774. 1

[2] C. Cinar, On the Positive Solutions of the Difference Equation $x_{n+1}=a x_{n-1} /\left(1+b x_{n} x_{n-1}\right)$, Appl. Math. Comput., 156 (2004), 587-590.

[3] C. Cinar, On the Positive Solutions of the Difference Equation $x_{n+1}=x_{n-1} /\left(1+x_{n} x_{n-1}\right)$, Appl. Math. Comput., 150 (2004), 21-24.

[4] C. Cinar, On the Positive Solutions of the Difference Equation $x_{n+1}=x_{n-1} /\left(-1+x_{n} x_{n-1}\right)$, Appl. Math. Comput., 158 (2004), 813-816.

[5] C. Cinar, On the Positive Solutions of the Difference Equation $x_{n+1}=x_{n-1} /\left(-1+a x_{n} x_{n-1}\right)$, Appl. Math. Comput., 158 (2004), 793-797.

[6] C. Cinar, On the Positive Solutions of the Difference Equation $x_{n+1}=x_{n-1} /\left(1+a x_{n} x_{n-1}\right)$, Appl. Math. Comput., 158 (2004), 809-812.

[7] E. M. Elsayed, On the Difference Equation $x_{n-5} /\left(-1+x_{n-2} x_{n-5}\right)$, Int. J. Contemp. Math. Sci., 33 (2008), 1657-1664.

[8] E. M. Elsayed, On the solutions and periodic nature of some systems of difference equations, Int. J. Biomath., 7 (2014), 26 pages.

[9] E. M. Elsayed, On the Solution of Some Difference Equations, Eur. J. Pure Appl. Math., 3 (2011), 287-303. 1, 1, 4.1.1, 4.1.2, $4.2 .1,4.2 .2,5$

[10] E. M. Elsayed, Solutions of Rational Difference System of Order Two, Math. Comput. Modelling, 55 (2012), 378-384.

[11] E. M. Elsayed, Solution and Attractivity for a Rational Recursive Sequence, Discrete Dyn. Nat. Soc., 2011 (2011), 17 pages. 1

[12] G. Feng, X. J. Yang, H. M. Srivasta, Exact traveling-wave solutions for linear and nonlinear heat-transfer equations, Thermal Science, 21 (2017), 2307-2311. 1

[13] M. Folly-Gbetoula, Symmetry, reductions and exact solutions of the difference equation $u_{n+2}=\left(a u_{n}\right) /\left(1+b u_{n} u_{n+1}\right)$, J. Difference Equ. Appl., 23 (2017), 1017-1024. 1

[14] M. Folly-Gbetoula, A. H. Kara, Symmetries, conservation laws, and Integrability of Difference Equations, Adv. Difference Equ., 2014 (2014), 14 pages.

[15] M. Folly-Gbetoula, A. H. Kara, The invariance, Conservation laws and Integration of some Higher-order Difference Equations, Advances and Applications in Discrete Mathematics, 18 (2017), 71-86. 1

[16] P. E. Hydon, Difference Equations by Differential Equation Methods, Cambridge University Press, Cambridge, (2014). $1,1.1$

[17] Y. Ibrahim, On the Global Attractivity of Positive Solutions of a Rational Difference Equation, Selcuk J. Appl. Math., 9 (2008), 3-8. 1

[18] T. F. Ibrahim, On the Third Order Rational Difference Equation $x_{n+1}=x_{n} x_{n-2} / x_{n-1}\left(a+b x_{n} x_{n-2}\right)$, Int. J. Contemp. Math. Sci., 27 (2009), 1321-1334. 1

[19] D. Levi, L. Vinet, P. Winternitz, Lie group Formalism for Difference Equations, J. Phys. A, 30 (1997), 633-649. 1

[20] G. R. W. Quispel, R. Sahadevan, Lie Symmetries and the Integration of Difference Equations, Phys. Lett. A, 184 (1993), 64-70. 1

[21] X. J. Yang, F. Gao, H. M. Srivasta, Exact travelling wave solutions for local fractional two-dimensional Burgers-type equations, Comput. Math. Appl., 26 (2017), 203-210. 1

[22] X. J. Yang, J. A. T. Machado, D. Baleanu, Exact traveling-wave solution for local fractional Boussinesq equation in fractal domain, Fractals, 25 (2017), 7 pages.

[23] X. J. Yang, J. A. T. Machado, D. Baleanu, C. Cattani, On exact traveling-wave solutions for local fractional Korteweg-de Vries equation, Chaos, 26 (2016), 5 pages. 1 